\title{
Detection of Positive and Negative Ions from a Flowing Atmospheric Pressure Afterglow Using a Mattauch-Herzog Mass Spectrograph Equipped with a Faraday-Strip Array Detector
}

\author{
Gregory D. Schilling, a Jacob T. Shelley, James H. Barnes IV, \\ Roger P. Sperline, ${ }^{\mathrm{c}}$ M. Bonner Denton, ${ }^{\mathrm{c}}$ Charles J. Barinaga, ${ }^{\mathrm{d}}$ \\ David W. Koppenaal, ${ }^{\mathrm{d}}$ and Gary M. Hieftje ${ }^{\mathrm{a}}$ \\ a Department of Chemistry, Indiana University, Bloomington, Indiana, USA \\ ${ }^{b}$ University Multispectral Laboratories, Ponca City, Oklahoma, USA \\ ${ }^{\mathrm{c}}$ Department of Chemistry, University of Arizona, Tucson, Arizona, USA \\ d Pacific Northwest National Laboratory, Richland, Washington, USA
}

An ambient desorption/ionization (ADI) source, known as the flowing atmospheric pressure afterglow (FAPA), has been coupled to a Mattauch-Herzog mass spectrograph (MHMS) equipped with a focal plane camera (FPC) array detector. The FAPA ionization source enables direct mass spectral analysis of solids, liquids, and gases through either positive or negative ionization modes. In either case, spectra are generally simple with dominant peaks being the molecular ions or protonated molecular ions. Use of the FAPA source with the MHMS allows the FPC detector to be characterized for the determination of molecular species, whereas previously only atomic mass spectrometry (MS) has been demonstrated. Furthermore, the FPC is shown to be sensitive to negative ions without the need to change any detector parameters. The analysis of solid, liquid, and gaseous samples through positive and negative ionization is demonstrated with detection limits (1-25 fmol/s, $\sim 0.3-10 \mathrm{pg}$ of analyte per $\mathrm{mL}$ of helium) surpassing those obtained with the FAPA source coupled to a time-of-flight mass analyzer. (J Am Soc Mass Spectrom 2010, 21, 97-103) (c) 2010 American Society for Mass Spectrometry

A novel ionization source and detector array have been coupled to a Mattauch-Herzog mass spectrograph (MHMS). The ionization source, known as the flowing atmospheric-pressure afterglow (FAPA), can directly desorb and ionize analytes from their native states. The array detector, termed the focal plane camera (FPC), enables the simultaneous collection of a range of mass-to-charge $(\mathrm{m} / \mathrm{z})$ values.

The FAPA ionization source consists of a glow discharge in helium using a pin-to-plate configuration. A hole at the center of the plate electrode enables the flow of helium ions and metastables $\left(\mathrm{He}^{*}\right)$ from the discharge cell into the open atmosphere. These energetic species ionize atmospheric constituents, including water, oxygen, and nitrogen, which in turn can ionize, through chemical ionization [1], analytes from samples placed in the afterglow region. As with other ambient desorption/ionization (ADI) sources, solids, liquids, or gases can be analyzed with the FAPA source, and spectra are generally very simple [1-3]. Additionally,

Address reprint requests to Dr. G. M. Hieftje, Department of Chemistry, Indiana University, Bloomington, IN, 47405, USA. E-mail: hieftje@indiana. edu positive and negative ions can be generated with the ionization source.

The FPC utilizes a linear arrangement of hundreds of Faraday strips. Traditionally, Faraday-based detectors have been inferior in sensitivity to ion-multiplying devices because of their lack of direct amplification. However, the use of Faraday detectors continued because of the superior precision they offer. With the FPC, high precision is retained while sensitivity is boosted by incorporating electronic amplification within each detection channel. Furthermore, because the Faraday array detector is based exclusively on charged particle collection, the mass-bias effects observed with energybased detectors is not a problem.

Previously, the FPC has been used exclusively for atomic mass spectrometry [4-10]. However, three notable FPC traits make it attractive for molecular analyses. First, as just stated, mass bias should not be problematic with the Faraday-based FPC. Second, since the FPC merely integrates charge, enhanced sensitivity should be observed for analytes that carry multiple charges. This advantage would be most significant through the use of an ESI source, which often generates multiply charged species. A final trait of the FPC is its ability 
to integrate either positive or negative ions without changing any operational parameters within the detector. In the current manuscript, results from the coupling of the FAPA source to a Mattauch-Herzog mass spectrograph (MHMS) fitted with FPC detection are presented. This marriage permits characterization of the FPC device for the detection of both positive and negative molecular ions.

\section{Experimental}

\section{FAPA}

The design of the FAPA source has been described previously $[1,2,11]$, so only a brief description and information pertinent to the current work will be presented here. A schematic of the FAPA ionization source is shown in Figure 1. The FAPA source consists of a cylindrical cell that holds two electrodes. The electrodes are a tungsten pin that has been filed to a point and a brass plate that incorporates a $1.25-\mathrm{mm}$ orifice. Included in the brass plate electrode is a nipple that surrounds the orifice and provides an anchor point for the discharge. The cylindrical cell was made of either Teflon (for positive ion mode) or glass (for negative ion mode) and contains a gas inlet port through which a flow of 1-3 L/min of helium (99.999\% ultra high purity, Airgas, Radnor, PA) was introduced. A discharge is created in the atmospheric pressure He by applying a voltage of 400-500 V between the two electrodes by means of a high voltage power supply (EH Series; Glassman High Voltage, Inc., Whitehouse Station, NJ). The power supply was isolated from ground by an isolation transformer (SIT 30-500; Stangenes Industries, Inc., Palo Alto, CA, USA). This arrangement allowed the discharge to be referenced to a potential other than ground, which was necessary for efficient ion sampling into the MHMS. For positive ion detection, the pin electrode was held at a potential more positive than the plate, whereas for the negative ion mode the reverse was necessary for reasons of ion sampling.

\section{Sample Introduction}

Samples were introduced into the FAPA source by several alternative methods. First, gas samples were introduced through a glass capillary as described previously [12]. Additionally, for the gas samples, a 100$\mathrm{mL}$, three-neck round-bottom flask was used as a stirred exponential dilution chamber for quantitative work. This method has also been described previously [1]. The vapor from the dilution chamber was introduced into the FAPA by means of the above-mentioned glass capillary. Second, solid and liquid samples were introduced by either the use of a sampling shelf affixed to the MHMS interface or a glass probe held by a Teflon spacer attached to the MHMS interface. Both of these methods have previously been used with the FAPA source [2].

\section{MHMS}

The MHMS has been described previously [4, 13, 14]. Changes made to the MHMS to obtain efficient ion sampling from the FAPA ionization source have also been described [12]; however, a diagram of the ion sampling interface is shown in Figure 1, which includes an inset plot showing the potential profile used in the positive ionization mode. The use of our MHMS for the detection of negative ions has never been reported. To achieve negative-ion mass detection with the MHMS, all ion-optic potentials, including the polarity of the accelerating potential and the direction of the magnetic field, were inverted. This inversion was accomplished by switching the polarity on six high-voltage power supplies (one SL-30 and five
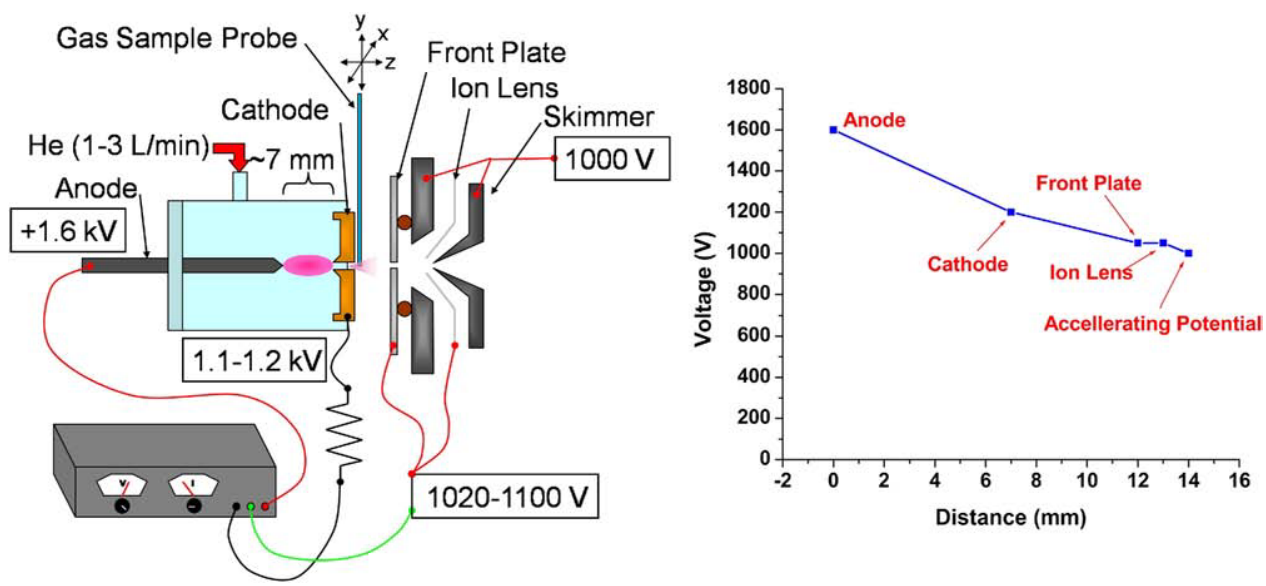

Figure 1. Diagram of the FAPA and MHMS ion-sampling interface in positive ionization mode. The power supply to control the FAPA source was referenced to the front plate of the MHMS for efficient ion sampling. The inset plot shows the potential profile starting from the anode and ending with the MHMS acceleration potential. 

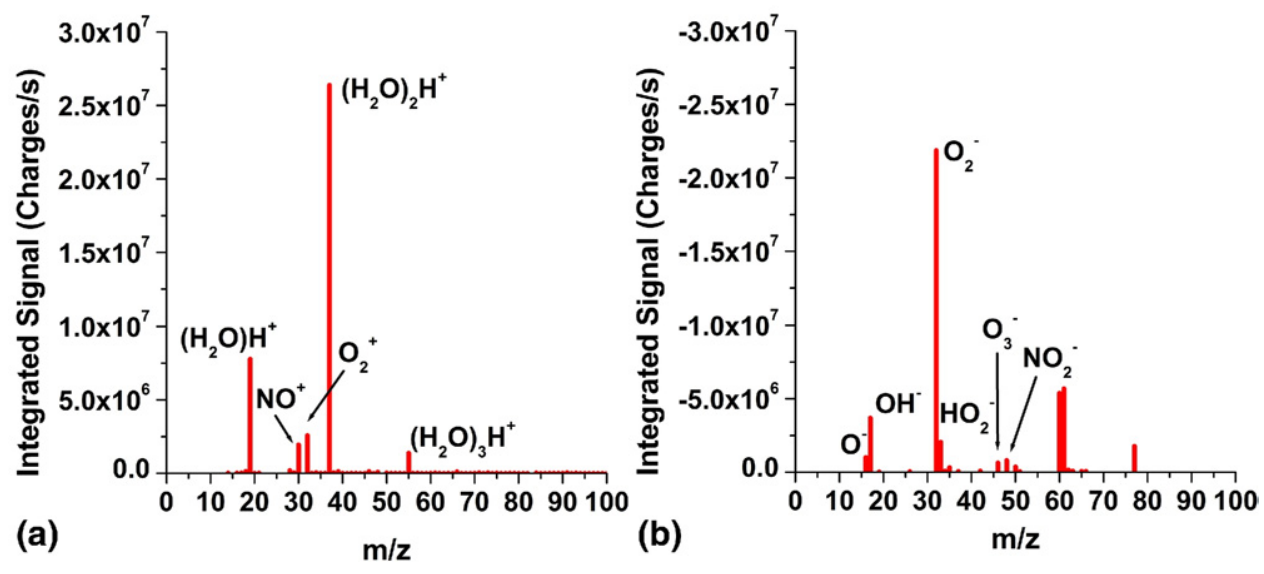

Figure 2. Background spectra (shown as stick plots) for the FAPA-MHMS-FPC system operating in (a) positive-ion mode using a Teflon-bodied cell, and (b) negative-ion mode using a glass-bodied cell. The total ion current in both cases is about the same.

SL-10; Spellman High Voltage Electronics Corp., Plainville, NY, USA) and reversing the current through the electromagnet coils.

\section{FPC}

A previously described 128-channel Faraday-strip array detector [9] was used to collect all of the data presented here. This detector incorporates pixels of $45-\mu \mathrm{m}$ width placed on $50-\mu \mathrm{m}$ centers, which are tall enough to span the entire height of the magnet gap in the MHMS instrument $(6.35 \mathrm{~mm})$. Each collector uses its own capacitive trans-impedance amplifier (CTIA), which features two individually selectable levels of gain. No changes in the detector were necessary to collect negative ions because each CTIA is biased in a way to integrate either positively or negatively depending on the polarity of the charge that is collected. No changes to the previously described [9] detector operation were made.

\section{Reagents and Samples}

A wide range of sample types, including gases, liquids, and solids, were used for the following experiments. In all cases, reagents were analytical grade. For gas analysis, vapors of acetone (Mallinckrodt Baker, Inc., Phillipsburg, NJ, USA), ethanol (Pharmco Products, Inc., Brookfield, CT, USA), and tetrahydrofuran (Mallinckrodt Baker, Inc.) were used. Liquid samples included dichloromethane, trinitrotoluene in acetonitrile (Restek Corp., Bellefonte, PA, USA), and m-nitrophenol (Sigma-Aldrich, St. Louis, MO, USA) dissolved in acetonitrile (Fisher Scientific, Pittsburgh, PA, USA). Caffeine (Matheson Coleman and Bell, Norwood, OH, USA), freshly ground coffee beans (Millstone; J. M. Smucker Company, Orrville, $\mathrm{OH}$, USA), and a tablet containing diflunisal were used for solid samples. All samples were introduced directly into the afterglow of the FAPA source according to the sample introduction methods described above.

\section{Results and Discussion}

\section{Signal Optimization}

Major signal optimization efforts were focused on the detection of positive ions, as this is the normal operational mode of the MHMS. The key factor for signal optimization was found to be discharge and samplinginterface potentials. When the FAPA source was first coupled to the MHMS-FPC instrument, no ions were observed. In this first case, the glow discharge used to create the flowing afterglow was referenced to ground potential, i.e., the cathode was held at $0 \mathrm{~V}$. This grounded cathode was placed a short distance (2-10 $\mathrm{mm}$ ) from the sampling interface of the MHMS, which was held at 1000-1150 V. The positive ions generated in this region could not overcome this large potential difference to be efficiently sampled into the MHMS. Consequently, the FAPA ionization source was electrically referenced to the front plate of the spectrograph. This created a field from high potential to low potential from the anode, through the cathode, and into the MHMS, while maintaining the discharge characteristics used in previous experiments [1].

Table 1. Sensitivities and detection limits for organic solvent vapors obtained with the FAPA-MHMS-FPC instrument

\begin{tabular}{lccc}
\hline & $\begin{array}{c}\text { Sensitivity } \\
(\text { counts/fmol/s) })^{\mathrm{a}}\end{array}$ & $\begin{array}{c}\text { Limit of } \\
\text { detection } \\
\text { MHMS-FPC } \\
(\mathrm{fmol} / \mathrm{s})^{\mathrm{a}}\end{array}$ & $\begin{array}{c}\text { Limits of } \\
\text { detection } \\
\text { TOFMS } \\
(\mathrm{fmol} / \mathrm{s})^{\mathrm{b}}\end{array}$ \\
\hline \hline Acetone & 101 & 0.9 & 9.5 \\
THF & 10 & 24 & - \\
Ethanol & 37 & 13 & - \\
\hline
\end{tabular}

aThis study.

bTOFMS from Reference [1]. 


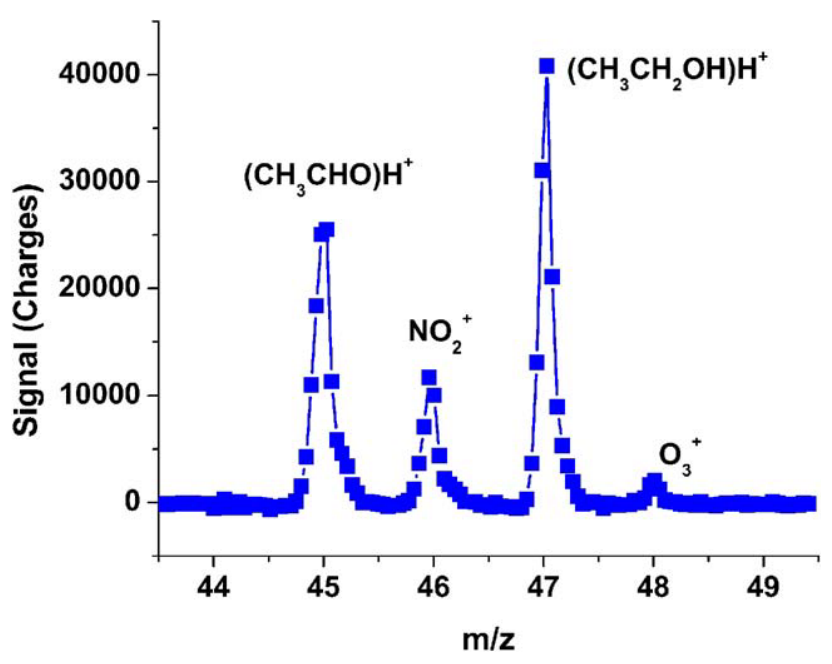

Figure 3. Mass spectrum of ethanol vapor showing the protonated molecular ion and a protonated fragment ion. Peaks at $m / z 46$ and 48 are from background ions. An exponential dilution experiment was used to generate a calibration curve and a $13 \mathrm{fmol} / \mathrm{s}$ detection limit was calculated for ethanol.

For the negative ion studies, it was necessary only to reverse the polarity of the discharge since the MHMS accelerating potential and all ion optic potentials were reversed. All other signal optimization was performed by fine tuning the ion optic potentials to obtain the highest reagent ion (protonated water clusters in positive ion mode and $\mathrm{O}_{2}{ }^{-}$in negative ion mode) signals.

\section{Background Spectra}

Background spectra were collected in both positive- and negative-ion modes and are shown in Figure $2 a$ and $b$, respectively. In both cases, the background spectra are shown as bar graphs generated from data taken in several mass windows. Therefore, no resolution information can be abstracted from these plots. However, all peaks were baseline-resolved, and the peak area was used to determine the height of the bars in the graphs. This process was employed because the total length of the present FPC enables the simultaneous acquisition of only 1-30 $\mathrm{m} / \mathrm{z}$ values, depending on the spectral region of observation. In the positive ionization mode, many of the same background peaks as previously reported with the FAPA source coupled to a time-of-flight mass analyzer [1] were recorded. The major components include water clusters, which are used as reagent ions for chemical ionization of analyte species. In addition to the water clusters, atmospheric components, such as $\mathrm{N}_{2}{ }^{+}, \mathrm{NO}^{+}, \mathrm{O}_{2}{ }^{+}$, and $\mathrm{NO}_{2}{ }^{+}$, were observed.

The negative ion background was first collected by using the Teflon-bodied cell. In this case, the vast majority of the peaks were attributable to clusters containing fluorine. Accordingly, the Teflon cell was replaced by a glass cell and the background spectrum of Figure $2 \mathrm{~b}$ was obtained. As expected, this spectrum was quite different from the positive-ion spectrum. The total ion current, however, was quite similar between the positive and negative background spectra. The major background component in the negative-ion mode is $\mathrm{O}_{2}{ }^{-}$. Additionally, other clusters containing oxygen or silicon also appear in the background, although no means of verification were applied. Little work on the fundamentals of negative ionization with the FAPA source has been completed; however, the likely mode of ionization is through charge exchange with these oxygenated ions, as is often the case in chemical ionization methods [15].

\section{Vapors}

The analysis of gaseous samples with the FAPAMHMS-FPC system has been achieved previously through the use of hydride generation [12]. However, for direct comparison with previous work, the quanti-
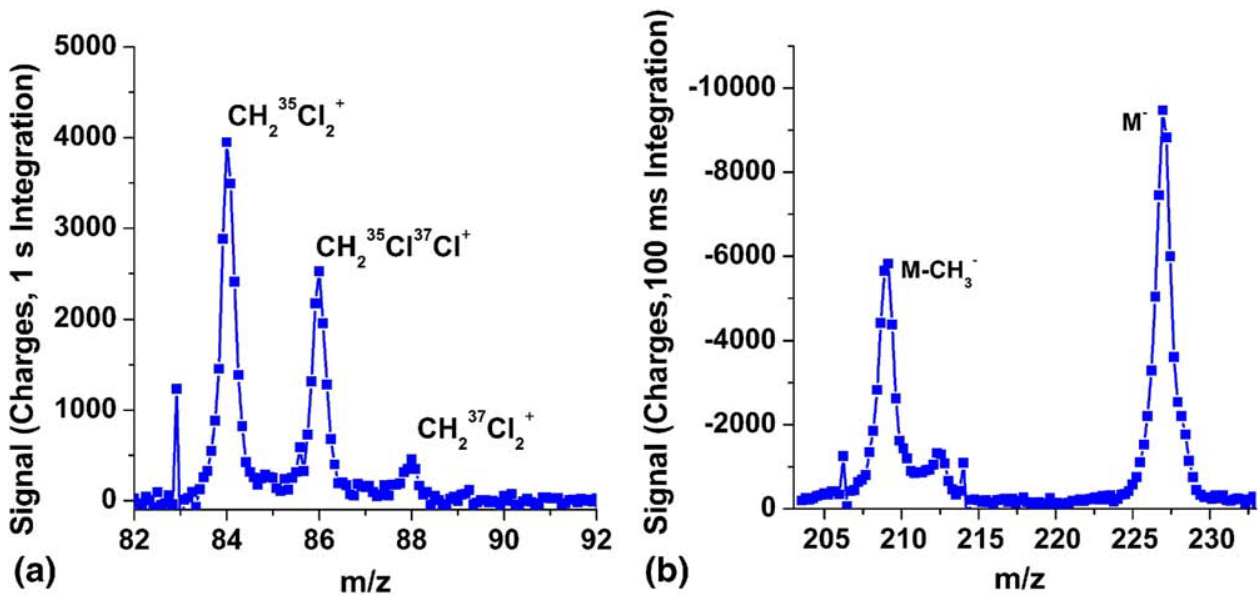

Figure 4. Detection of organic liquids with the FAPA-MHMS-FPC system. (a) Positive-ion spectrum obtained from dichloromethane placed directly on the tip of a wooden sample probe and analyzed in the FAPA source, and (b) negative-ion spectrum obtained from $500 \mathrm{~nL}$ of $1000 \mathrm{ppm}$ TNT in acetonitrile injected onto the wooden end of a Q-tip and placed in the afterglow. 
Table 2. Isotope ratio accuracy for dichloromethane

\begin{tabular}{cccc}
\hline Mass ratio & Experimental & Theoretical & \% Error \\
\hline \hline $84 / 86$ & 1.59 & 1.55 & 3 \\
$84 / 88$ & 9.62 & 9.52 & 1 \\
$86 / 88$ & 6.03 & 6.16 & 2
\end{tabular}

fication of organic vapors was performed here through the use of exponential dilution. In all cases, positive ion detection was used and the protonated molecular ion was monitored. Sensitivities and detection limits for the FAPA-MHMS-FPC system are compiled in Table 1. These values are about 10 times better than those reported for the FAPA source on a TOFMS instrument [1]. The reason for the improvement is probably the 10 times higher duty factor of the MHMS-FPC instrument over the TOFMS. A representative mass spectrum of ethanol vapor is shown in Figure 3. In this case, the vapor was swept from the dilution chamber via a stream of argon. To generate the calibration curve, time $(t)$ is converted to concentration $(C)$ by means of eq 1 , where $C_{0}$ is the initial analyte concentration in the chamber, $F$ is the gas-flow rate of the dilution gas, and $V$ is the volume of the chamber. The calibration curve shows linearity across three orders of magnitude, and a limit of detection of $15 \mathrm{fmol} / \mathrm{s}$ was calculated. The signal at the detection limit would correspond to a steady-state concentration of $3.6 \mathrm{pg} / \mathrm{mL}$ of ethanol in helium at $\sim 1 \mathrm{~atm}$ and $150-200{ }^{\circ} \mathrm{C}$.

$$
C=C_{0} e\left(\frac{F}{V} \cdot t\right)
$$

\section{Liquids}

Analysis of liquids is also possible with the FAPA ionization source and was achieved by dipping or applying the liquid to either a melting point tube or the wooden end of a Q-tip and placing the sample probe into the FAPA afterglow. Two example spectra are shown in Figure 4. The first (Figure 4a) shows the positive-ion spectrum obtained from introduction of dichloromethane into the afterglow region. The expected isotopic pattern is observed for the chlorine isotopes. The ratios of the peaks are compared in Table 2 with the theoretical ratios based on the elemental isotopic distributions. Errors of 1\%-3\% were obtained. Figure $4 \mathrm{~b}$ shows a negative-ion spectrum containing the molecular ion of trinitrotoluene (TNT). Loss of the methyl group is observed, showing that some fragmentation can occur. This spectrum was generated by placing $500 \mathrm{~nL}$ of a $1000 \mathrm{ppm}$ TNT in acetonitrile solution directly onto the end of a wooden applicator. The applicator was placed in the afterglow, and several $100 \mathrm{~ms}$ integrations were acquired. Additionally, solutions of 10-1000 ppm m-nitrophenol in acetonitrile were analyzed by dipping a wooden applicator into the solution for $5 \mathrm{~s}$ and then placing the applicator into the afterglow of the FAPA source. These data were used to generate a calibration curve, and a $0.9 \mathrm{ppm}$ limit of detection was calculated.

\section{Solids}

Perhaps the most useful type of analysis that can be performed with ADI-MS is the detection of analytes directly from the solid-state. The analysis of solid samples with the FAPA source has already been demonstrated [2]; a similar analysis performed with the current setup is illustrated in Figure 5, which shows spectra of both solid caffeine and a ground coffee been, obtained under identical instrumental conditions. Again, due to the limited FPC width, only the protonated molecular ion is shown. However, with both the MHMS-FPC instrument and a LECO Unique TOFMS instrument (LECO Corporation, Saint Joseph, MI, USA), caffeine with water cluster additions were observed. No attempts were made here to reduce the abundance of these caffeine water clusters, but future studies will be aimed in this direction to simplify the mass spectra, reduce the likelihood of spectral interfer-
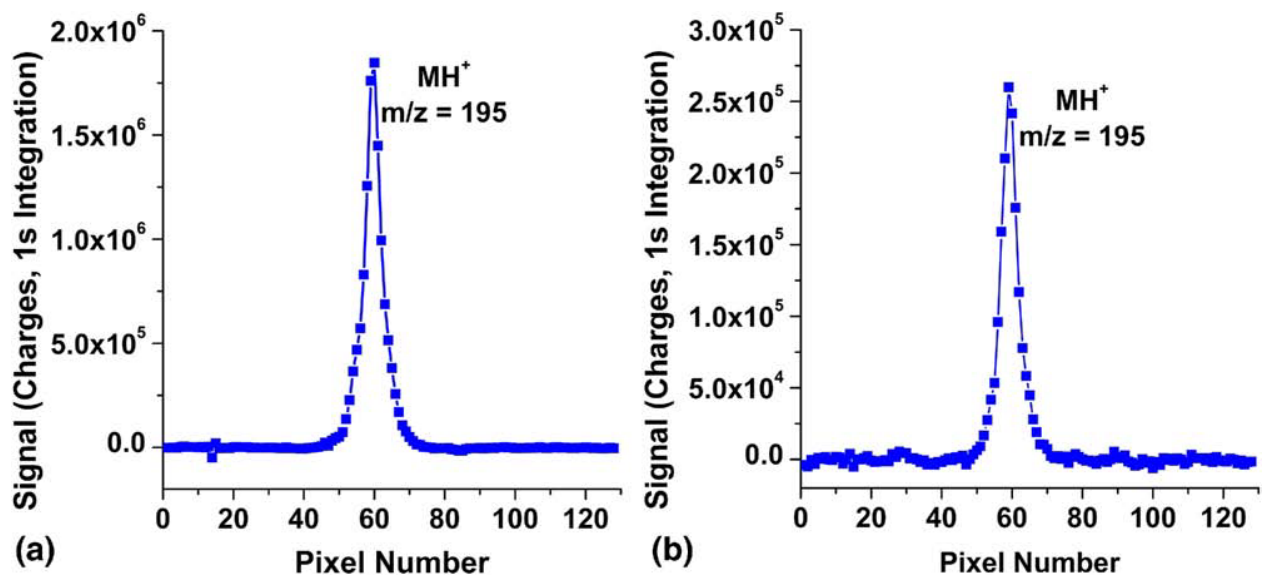

Figure 5. Positive-ion spectra of (a) solid caffeine, and (b) caffeine in a ground coffee bean, obtained using Q-tips as sampling probes. 

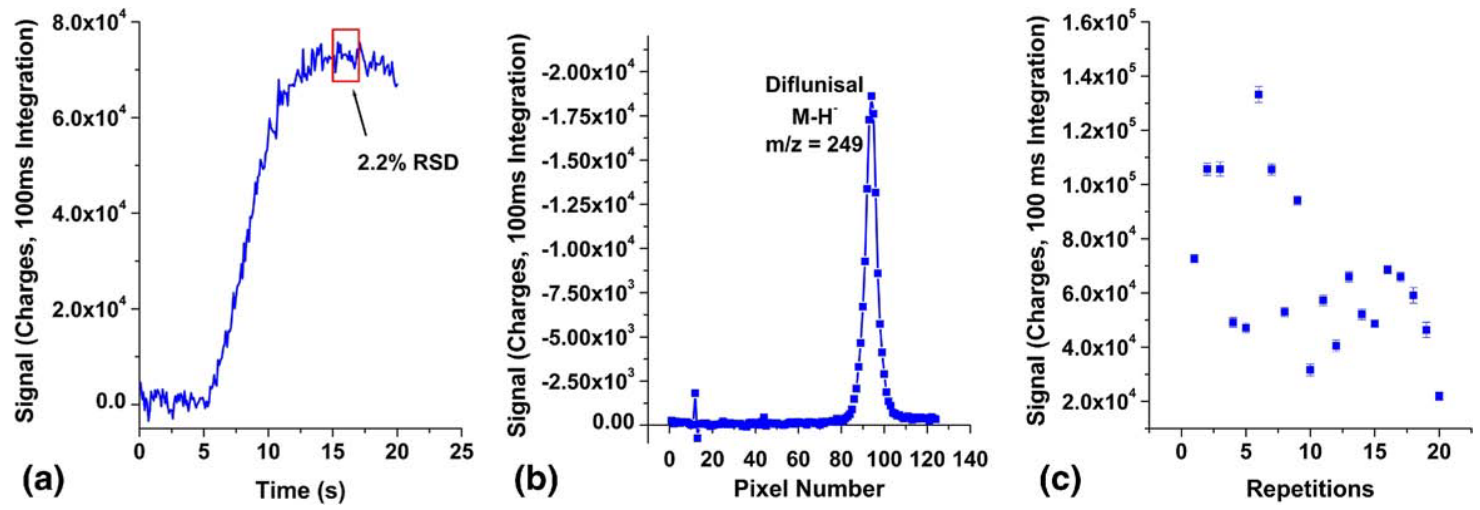

Figure 6. Analysis of diflunisal from a pharmaceutical tablet. (a) Transient produced from placing the tablet in the afterglow, (b) spectrum from the plateau region of the transient, and (c) 20 repetitions of placing material in the afterglow. Notice that the precision within a single repetition is about $2 \%$ RSD (a); however, precision degrades to $43 \%$ RSD (c) between runs, indicating the need for a more reproducible sample introduction method.

ences in more complicated samples, and improve the signal-to-noise ratio of the molecular ion peak. As would be expected, the two spectra in Figure 5 are nearly identical, differing only in peak height.

Negative-ion analysis of solid samples is also achievable with the FAPA-MHMS-FPC instrument. This capability is demonstrated in Figure 6, which shows a transient sample introduction curve, spectrum, and reproducibility for the direct analysis of a diflunisal pharmaceutical tablet. The material was sampled onto the wooden end of a Q-tip and then introduced into the FAPA source. The resulting transient signal is shown in Figure 6a. The wash-in time takes about $5-7 \mathrm{~s}$ and is followed by a signal plateau that continues for about $5 \mathrm{~s}$. The reproducibility within this plateau region is generally around $2 \% \mathrm{RSD}$, showing that desorption and ionization of solid material is quite stable. However, when the reproducibility of separate analyses is evaluated, as in Figure $6 c$, the precision degrades to $40 \%$ RSD or more. Reproducible sample introduction is obviously an area that must be addressed in ADI-MS.

\section{Conclusions}

Use of the FPC for the sensitive, accurate, and precise analysis of atomic species over very broad concentration ranges has been demonstrated previously [4, 9]. The current results exhibit the use of the FPC array detector for the detection of molecular species in both positive and negative ionization modes through the use of a new FAPA ionization source. Although not implemented in this study, automated mode switching should be quite simple and probably achievable within a single transient analysis. Furthermore, the sensitivity of the system was shown to be better than a commercial TOFMS system. The resolution achieved with the MHMS instrument system was somewhat disappointing; however, this limitation is most likely attributable to the MHMS and not the FPC, as the MHMS was designed for atomic analyses. Future ex- periments with the FPC and molecule ions should include the use of an electrospray ionization source. Because the FPC is a charge-integrating device, increased sensitivity should be observed for multiply charged ions.

Additionally, the present study demonstrates the successful coupling of the new FAPA source with a sector-field instrument with a large interface accelerating potential. This coupling has been achieved previously but only for gas analysis with a fixed sample inlet probe. While the FAPA source is shown to be very versatile, some deficiencies include cluster formation with background species and irreproducible sample introduction. Continued work is necessary in these areas.

\section{Acknowledgments}

The authors acknowledge support in part for this work by the U.S. Department of Energy, Office of Nonproliferation Research and Engineering. Pacific Northwest National Laboratory is operated by Battelle Memorial Institute for the Department of Energy under Contract DE-AC06-76RLO-1830. The authors also acknowledge support in part also by Prosolia, Inc. and by the Indiana METACyt Initiative of Indiana University, funded in part through a major grant from the Lilly Endowment, Inc.

\section{References}

1. Andrade, F. J.; Shelley, J. T.; Wetzel, W. C.; Webb, M. R.; Gamez, G.; Ray, S. J.; Hieftje, G. M. Atmospheric Pressure Chemical Ionization Source. 1. Ionization of Compounds in the Gas Phase. Anal. Chem. 2008, 80, 2646-2653.

2. Andrade, F. J.; Shelley, J. T.; Wetzel, W. C.; Webb, M. R.; Gamez, G.; Ray, S. J.; Hieftje, G. M. Atmospheric Pressure Chemical Ionization Source. 2. Desorption-Ionization for the Direct Analysis of Solid Compounds. Anal. Chem. 2008, 80, 2654-2663.

3. Shelley, J. T.; Ray, S. J.; Hieftje, G. M. Laser Ablation Coupled to a Flowing Atmospheric Pressure Afterglow for Ambient Mass Spectral Imaging. Anal. Chem. 2008, 80, 8308-8313.

4. Barnes, J. H.; Schilling, G. D.; Sperline, R.; Denton, M. B.; Young, E. T.; Barinaga, C. J.; Koppenaal, D. W.; Hieftje, G. M. Characterization of a Focal Plane Camera Fitted to a Mattauch-Herzog Geometry Mass Spectrograph. 2. Use with an Inductively Coupled Plasma. Anal. Chem. 2004, 76, 2531-2536.

5. Barnes, J. H., IV; Schilling, G. D.; Hieftje, G. M.; Sperline, R. P.; Denton, M. B.; Barinaga, C. J.; Koppenaal, D. W. Use of a Novel Array Detector 
for the Direct Analysis of Solid Samples by Laser Ablation Inductively Coupled Plasma Sector-Field Mass Spectrometry. I. Am. Soc. Mass Spectrom. 2004, 15, 769-776.

6. Barnes, J. H., IV; Schilling, G. D.; Sperline, R. P.; Denton, M. B.; Young, E. T.; Barinaga, C. J.; Koppenaal, D. W.; Hieftje, G. M. Coupling of a Gas Chromatograph to a Simultaneous-Detection Inductively Coupled Plasma Mass Spectrograph for Speciation of Organohalide and Organometallic Compounds. J. Anal. At. Spectrom. 2004, 19, 751-756.

7. Barnes, J. H., IV; Schilling, G. D.; Stone, S. F.; Sperline, R. P.; Denton, M. B.; Young, E. T.; Barinaga, C. J.; Koppenaal, D. W.; Hieftje, G. M. Simultaneous Multichannel Mass-Specific Detection for High-Performance Liquid Chromatography Using an Array Detector Sector-Field Mass Spectrometer. Anal. Bioanal. Chem. 2004, 380, 227-234.

8. Barnes, J. H., IV; Sperline, R.; Denton, M. B.; Barinaga, C. J.; Koppenaal, D.; Young, E. T.; Hieftje, G. M. Characterization of a Focal Plane Camera Fitted to a Mattauch-Herzog Geometry Mass Spectrograph. 1. Use with a Glow-Discharge Source. Anal. Chem. 2002, 74, 5327-5332.

9. Schilling, G. D.; Andrade, F. J.; Barnes, J. H.; Sperline, R. P.; Denton, M. B.; Barinaga, C. J.; Koppenaal, D. W.; Hieftje, G. M. Characterization of a Second-Generation Focal-Plane Camera Coupled to an Inductively Coupled Plasma Mattauch-Herzog Geometry Mass Spectrograph. Anal. Chem. 2006, 78, 4319-4325.
10. Schilling, G. D.; Andrade, F. J.; Barnes, J. H., IV; Sperline, R. P.; Denton, M. B.; Barinaga, C. J.; Koppenaal, D. W.; Hieftje, G. M. Continuous Simultaneous Detection in Mass Spectrometry. Anal. Chem. 2007, 79, 7662-7668.

11. Andrade, F. J.; Wetzel, W. C.; Chan, G. C. Y.; Webb, M. R.; Gamez, G.; Ray, S. J.; Hieftje, G. M. A New, Versatile, Direct-Current Helium Atmospheric-Pressure Glow Discharge. J. Anal. At. Spectrom. 2006, 21, 1175-1184.

12. Schilling, G. D.; Shelley, J. T.; Broekaert, J. A. C.; Sperline, R. P.; Denton, M. B.; Barinaga, C. J.; Koppenaal, D. W.; Hieftje, G. M. Use of an Ambient Ionization Flowing Atmospheric-Pressure Afterglow Source for Elemental Analysis Through Hydride Generation. I. Anal. At. Spectrom. 2008, 24, 34-40.

13. Barnes, J. H., IV; Schilling, G. D.; Denton, M. B.; Koppenaal, D. W.; Hieftje, G. M. Development and Characterization of an Electrostatic Quadrupole Extraction Lens for Mass Spectrometry. J. Anal. At. Spectrom. 2003, 18, 1015-1018.

14. Burgoyne, T. W.; Hieftje, G. M.; Hites, R. A. Design and Performance of a Plasma-Source Mass Spectrograph. J. Am. Soc. Mass Spectrom. 1997, 8, 307-318.

15. Harrison, A. G. Chemical Ionization Mass Spectrometry, 2nd ed.; CRC Press: Boca Raton, FL, 1992; p. 208. 